\title{
Dependência micorrízica de variedades comerciais de mamoeiro(1)
}

\begin{abstract}
Aldo Vilar Trindade(2), José Oswaldo Siqueira ${ }^{(3)}$ e Florício Pinto de Almeida(4)
Resumo - O genótipo da planta é fator preponderante na resposta à micorriza. Avaliou-se, neste trabalho, a resposta a fósforo e o grau de dependência micorrízica de variedades de mamoeiro (Carica papaya L.). O experimento foi realizado em casa de vegetação da Embrapa-Centro Nacional de Pesquisa de Mandioca e Fruticultura, com quatro variedades, testando-se seis doses de P: 0, 20, 40, 80, $140,280 \mathrm{mg} \mathrm{dm}^{-3} \mathrm{e}$ três tratamentos fúngicos (um controle não-micorrizado e a inoculação de Glomus clarum ou Gigaspora margarita). Foram estimados os seguintes parâmetros: dose de P para máxima eficiência micorrízica; valor T'; benefício da aplicação do P; benefício micorrízico; efíciência simbiótica, e dependência micorrízica. Plantas não-micorrizadas apresentaram resposta quadrática ao $\mathrm{P}$ aplicado $\mathrm{e}$ as inoculadas seguiram modelo raiz-quadrático, com grande incremento de matéria seca da parte aérea nas doses baixas; o benefício de P foi maior nas variedades Sunrise Solo e Improved Sunrise Solo Line 72/12, enquanto as variedades Baixinho de Santa Amália e Tainung no 1 apresentaram benefício micorrízico proporcionalmente maior, resultando em maior eficiência simbiótica. As variedades de mamoeiro apresentaram a seguinte classificação quanto à dependência micorrízica: Baixinho de Santa Amália $=$ Tainung no $1>$ Sunrise Solo $=$ Improved Sunrise Solo - Line 72/12. A maior ou menor dependência relacionou-se com a capacidade da variedade em produzir raízes.
\end{abstract}

Termos para indexação: Carica papaya, inoculação, adubação fosfatada, absorção de nutrientes, resposta da planta.

\section{Mycorrhizal dependency of papaya commercial varieties}

Abstract - Plant genome is an important factor in plant response to arbuscular fungi. This work was designed to evaluate the degree of response to $\mathrm{P}$ application and mycorrhizal dependence of different varieties of papaya (Carica papaya L.). The experiment was performed under greenhouse conditions at Embrapa-Centro Nacional de Pesquisa de Mandioca e Fruticultura, Cruz das Almas, BA, Brazil, using seedlings of four varieties, six rates of P: 0, 20, 40, 80, 140 and $280 \mathrm{mg} \mathrm{dm}^{-3}$, and three fungi treatments (one control uninoculated and inoculation with Glomus clarum or Gigaspora margarita). The following parameters were estimated: rate of $\mathrm{P}$ for maximum mycorrhizal efficiency; $\mathrm{T}$ ' value; $\mathrm{P}$ benefit, mycorrhizal benefit, symbiotic efficiency, and mycorrhizal dependence. Control plants had a quadratic response to applied $\mathrm{P}$, while those inoculated had a root-quadratic response with a great increment in growth at lower rates; phosphorus benefit was higher in Sunrise Solo and Improved Sunrise Solo - Line 72/12 varieties, while the other two varieties showed a higher mycorrhizal benefit, resulting in a higher symbiotic efficiency. Papaya varieties showed the following classification of mycorrhizal dependency: Baixinho de Santa Amália $=$ Tainung no $1>$ Sunrise Solo $=$ Improved Sunrise Solo - Line 72/12. Mycorrhizal dependence related with the capability of the variety to produce roots. Index terms: Carica papaya, inoculation, phosphate fertilization, nutrient uptake, plant response.

(1) Aceito para publicação em 13 de março de 2001 . Extraído da Tese de Doutorado apresentada pelo primeiro autor à Universidade Federal de Lavras (Ufla), Lavras, MG

${ }^{(2)}$ Embrapa-Centro Nacional de Pesquisa de Mandioca e Fruticultura, Caixa Postal 7, CEP 44380-000 Cruz das Almas, BA E-mail: aldo@cnpmf.embrapa.br

(3)Ufla, Caixa Postal 37, CEP 37200-000 Lavras, MG. E-mail: siqueira@ufla.br

(4) Universidade Federal da Paraíba, Campus II, CEP 58102-970 Campina Grande, PB. E-mail: floricio@deag.ufpb.br

\section{Introdução}

Os fungos micorrízicos arbusculares (FMAs) ocorrem em mais de $90 \%$ das plantas terrestres, e têm como característica marcante a ubiqüidade nos ecossistemas. O grau de interação entre o fungo e a planta depende mais do genótipo da planta, cujo principal fator controlador da associação é o estado 
nutricional do vegetal. De acordo com o fungo considerado, a resposta da planta pode sofrer alterações, mas o seu potencial de resposta à colonização parece ser uma característica intrínseca, de herança genética (Janos, 1988), relacionado às características morfológicas, fisiológicas ou fenológicas do hospedeiro, que controlam a demanda e suprimento de $\mathrm{P}$, e, assim, o grau de dependência da planta (Koide, 1991). Com base nisto, o teor de $P$ no solo mais apropriado para a resposta à micorriza é altamente variável entre as espécies e genótipos, e há diferenças mesmo em plantas muito próximas geneticamente, como em progênies de mesma espécie (Clement \& Habte, 1995). Assim, as condições de solo e características do genótipo do hospedeiro como sistema radicular, taxa de crescimento e alocação de carboidratos (Manjunath \& Habte, 1991; Amijee et al., 1993) são componentes determinantes da formação e efeitos das micorrizas sobre as plantas.

Como as plantas apresentam graus de dependência naturalmente variáveis (Manjunath \& Habte, 1991; Declerck et al., 1995), os trabalhos de melhoramento também podem gerar genótipos com diferentes exigências nutricionais e capacidade de absorção. Admitindo-se que um dos grandes benefícios da colonização micorrízica é o aumento da absorção de nutrientes, é razoável esperar que existam diferenças genotípicas relativas à simbiose micorrízica. A seleção de cultivares ou de genótipos prontamente colonizados por fungos MA pode ser um passo importante no sentido de se chegar a uma menor dependência dos fertilizantes fosfatados. Plantas mais dependentes seriam um alvo mais apropriado para o emprego de um programa de inoculação que vise a redução das doses de $\mathrm{P}$ aplicadas ao solo.

Diversos trabalhos têm mostrado elevada eficiência micorrízica em espécies cítricas, café, e mandioca, entre outras (Sieverding, 1991; Oliveira et al., 1992; Saggin Júnior \& Siqueira, 1996). Em face da viabilidade técnica da inoculação de fungos na fase de produção de mudas, diversas culturas apresentam possibilidades para uso da inoculação. As fruteiras, de maneira geral, apresentam grande potencial, principalmente as que possuem sistema radicular pouco ramificado, com poucos pêlos radiculares, e crescimento rápido. $\mathrm{O}$ mamoeiro se enquadra nessas condições, apresentando possibilidades de aplicação de fungos MA, já que diversos trabalhos, desde o de Ramirez et al. (1975), demonstraram elevada resposta da planta à colonização, que pode atingir valores da ordem de 90\% (Silva \& Siqueira, 1991; Mohandas, 1992; Weber \& Amorim, 1994; Auler, 1995).

Este trabalho objetivou estudar a resposta de variedades comerciais de mamoeiro quanto à eficiência micorrízica, em diferentes doses de $\mathrm{P}$ no solo, e identificar o grau de dependência micorrízica dessas variedades.

\section{Material e Métodos}

O experimento foi realizado em casa de vegetação, nos meses de fevereiro e março de 1997, na Embrapa-Centro Nacional de Pesquisa de Mandioca e Fruticultura, com três variedades do grupo 'Solo': Sunrise Solo, Improved Sunrise Solo - Line 72/12, Baixinho de Santa Amália, e uma variedade do grupo 'Formosa': Tainung no 1 (híbrido de procedência de Taiwan). Para cada variedade, empregou-se um esquema fatorial composto por seis doses de P aplicadas ao solo: 0, 20, 40, 80, 140 e $280 \mathrm{mg} \mathrm{dm}^{-3}$, e três tratamentos fúngicos, a saber: um controle, e a inoculação de dois fungos pré-selecionados em experimento anterior - Glomus clarum e Gigaspora margarita -, totalizando 18 tratamentos. Foram empregadas quatro repetições em um delineamento de blocos casualizados, em que cada parcela foi representada por um saco de plástico preto com furos, com $1 \mathrm{dm}^{3}$ de solo e contendo uma planta.

Utilizou-se amostra de um Latossolo Amarelo-álico, textura franco-argilo-arenosa, coletado em área experimental da Embrapa-Centro Nacional de Pesquisa de Mandioca e Fruticultura, na profundidade de 10 a $30 \mathrm{~cm}$, apresentando as seguintes características químicas: capacidade máxima de adsorção de $\mathrm{P}, 229 \mu \mathrm{g} \mathrm{cm}^{-3} ; \mathrm{pH} \mathrm{4,4}$; $3 \mathrm{mg} \mathrm{dm}^{-3}$ de P; $31 \mathrm{mg} \mathrm{dm}^{-3}$ de K; $8 \mathrm{mmol}_{\mathrm{c}} \mathrm{dm}^{-3}$ de Ca; $4 \mathrm{mmol}_{\mathrm{c}} \mathrm{dm}^{-3} \mathrm{de} \mathrm{Mg} ; 8 \mathrm{mmol}_{\mathrm{c}} \mathrm{dm}^{-3} \mathrm{de} \mathrm{Al}$ e $1,61 \mathrm{~g} \mathrm{~kg}^{-1} \mathrm{de}$ matéria orgânica. Após peneiramento e fumigação (bromex, $393 \mathrm{~cm}^{3} \mathrm{~m}^{-3}$ de solo), fez-se a correção do $\mathrm{pH}$ do solo, aplicando-se o equivalente a $1,6 \mathrm{tha}^{-1}$ de calcário na forma de uma mistura de $\mathrm{CaCO}_{3}+\mathrm{MgCO}_{3}$, P.A. (proporção de 4:1 em mmol $_{\mathrm{c}}$ de $\mathrm{Ca}: \mathrm{Mg}$ ). $\mathrm{O} \mathrm{P}$ foi aplicado na forma de superfosfato triplo. Fez-se uma aplicação de $80 \mathrm{mg} \mathrm{dm}^{-3} \mathrm{de} \mathrm{K}(\mathrm{KCl})$. As características do solo após a calagem e adubação são mostradas na Tabela 1 .

As plântulas foram obtidas por pré-germinação, em mistura de areia + vermiculita $(1: 1)$ autoclavada. A inoculação foi realizada com solo-inóculo depositado em orifício central feito no recipiente de cultivo, sendo 
em seguida feita a repicagem da muda de mamoeiro, de forma que as raízes ficaram em contato íntimo com o inóculo. O fungo G. clarum foi obtido da Universidade Federal de Lavras, tendo sido isolado de plantios de café de Minas Gerais; G. margarita foi obtido junto à Empresa Maguari, do Município de Araguari, MG.

Adubações com N, S e micronutrientes foram realizadas em cobertura, via solução nutritiva, totalizando $30 \mathrm{mg} \mathrm{dm}^{-3}$ de N, 36,8 $\mathrm{mg} \mathrm{dm}^{-3}$ de $\mathrm{S}$, parcelados em cinco aplicações e $0,81 \mathrm{mg} \mathrm{dm}^{-3}$ de B, 3,6 $\mathrm{mg} \mathrm{dm}^{-3} \mathrm{de} \mathrm{Mn}$, $1,3 \mathrm{mg} \mathrm{dm}^{-3} \mathrm{de} \mathrm{Cu}, 3,9 \mathrm{mg} \mathrm{dm}^{-3}$ de Zn, $0,15 \mathrm{mg} \mathrm{dm}^{-3} \mathrm{de}$ Mo, e 1,6 $\mathrm{mg} \mathrm{dm}^{-3}$ de Fe em aplicação única, aos dez dias após a repicagem. Aos 41 dias após a repicagem, coletouse a parte aérea, para obtenção do peso de matéria seca (MSPA) e determinação dos teores de nutrientes Do sistema radicular foram coletadas todas as raízes, para avaliação do comprimento (Newman, 1966). Posteriormente, fez-se uma amostragem de raízes com vistas à coloração, e avaliação da colonização micorrízica total (Ambler \& Young, 1977).

Os dados de cada variedade em separado foram submetidos à análise de variância. Fez-se o desdobramento das doses de $\mathrm{P}$ dentro de cada tratamento fúngico, avaliando-se o efeito por análise de regressão. Com base nas curvas de resposta a $\mathrm{P}$ foram calculados os seguintes índices: 1) teor de $\mathrm{P}$ no solo para máxima resposta à micorriza; 2) valor T', dose de $P$ aplicada ao solo, acima da qual as plantas não-micorrizadas não crescem diferentemente das micorrizadas (ponto no gráfico onde há encontro das curvas do tratamento-controle e do tratamento micorrizado), e que representa a dependência micorrízica, de acordo com Janos (1988); quando não houve cruzamento, tomou-se a maior dose de $\mathrm{P}$ utilizada como referência; 3 ) benefício do $\mathrm{P}(\mathrm{BP})=$ área formada entre o eixo $\mathrm{X}($ doses de $\mathrm{P})$ e os pontos plotados pela equação de regressão do tratamento controle não-micorrizado (Saggin Júnior \& Siqueira, 1995); 4) benefício micorrízico (BM) = área formada pela diferença entre a curva do tratamento micorrizado e a do controle não-micorrizado (Saggin Júnior \& Siqueira, 1995). De posse dos valores de benefício micorrízico e do
P, calculou-se a eficiência simbiótica (ES) de cada fungo, de acordo com a expressão abaixo, sendo, esses fungos, enquadrados em categorias (Saggin Júnior \& Siqueira, 1995):

$\mathrm{ES}(\%)=100(\mathrm{BM} / \mathrm{BP})$.

\section{Resultados e Discussão}

Plantas sem inoculação apresentaram resposta quadrática, ocorrendo aumento gradual no crescimento, pelo aumento das doses de $\mathrm{P}$, com tendência de queda nas doses mais elevadas (Figura 1). Considerando-se estas plantas, a produção máxima de MSPA foi atingida com doses de P de 200, 215, 191 e $194 \mathrm{mg} \mathrm{dm}^{-3}$, respectivamente, no Sunrise Solo, no Improved Sunrise Solo, no Baixinho, e no Tainung n $\mathrm{o} 1$.

Em todas as variedades e plantas submetidas a inoculação, o acúmulo de MS da parte aérea, em decorrência das doses crescentes de $\mathrm{P}$, seguiu um modelo raiz-quadrático, o que evidencia resposta acentuada nas doses mais baixas de P. Este padrão permitiu que a demanda externa de $\mathrm{P}$ fosse reduzida nas plantas micorrizadas. Para ambos os fungos, a relação com a planta causou efeito depressivo na variedade Sunrise Solo quando da aplicação da dose máxima de $\mathrm{P}$. Como a capacidade máxima de adsorção de P (CMAP) do solo em estudo é relativamente baixa, o sistema se torna mais facilmente saturável com o uso de doses crescentes de $\mathrm{P}$, e o crescimento máximo pode ser atingido em doses menores. Com solos de maior CMAP, o mamoeiro pode responder a doses maiores que $280 \mathrm{mg} \mathrm{dm}^{-3}$ (Auler, 1995). Entretanto, nas grandes regiões produtoras da cultura predominam solos com elevados teores de areia (Trindade, 1998), o que sugere a aplicação de doses menores de $\mathrm{P}$.

Tabela 1. Caracterização química do solo após aplicação de calcário, fósforo e potássio.

\begin{tabular}{cccccccccccc}
\hline $\begin{array}{c}\text { Doses de } \mathrm{P} \\
\left(\mathrm{mg} \mathrm{dm}^{-3}\right)\end{array}$ & $\begin{array}{c}\mathrm{pH} \mathrm{em} \\
\mathrm{H}_{2} \mathrm{O}\end{array}$ & \multicolumn{2}{c}{$\mathrm{P}$} & $\begin{array}{c}\mathrm{K} \\
-\left(\mathrm{mg} \mathrm{dm}^{-3}\right)--\end{array}$ & $\begin{array}{c}\mathrm{Ca} \\
-----------------\mathrm{mmol}_{\mathrm{c}} \mathrm{dm}^{-3}\end{array}$ & $\mathrm{Mg}$ & $\mathrm{Al}$ & $\mathrm{CTC}$ & $\begin{array}{c}\mathrm{V} \\
(\%)\end{array}$ & $\begin{array}{c}\text { M.O. } \\
\left(\mathrm{g} \mathrm{kg}^{-1}\right)\end{array}$ \\
\hline 0 & 6,2 & 1 & 198 & 32 & 10 & 1 & 0,6 & 47,7 & 59,8 & 79,8 & 1,39 \\
20 & 6,2 & 11 & 174 & 40 & 10 & 0 & 0,8 & 55,3 & 68,5 & 80,7 & 1,36 \\
40 & 6,2 & 20 & 144 & 31 & 16 & 0 & 0,7 & 51,4 & 64,6 & 79,6 & 1,43 \\
80 & 6,0 & 41 & 180 & 32 & 13 & 0 & 0,8 & 50,4 & 64,7 & 77,9 & 1,36 \\
140 & 6,1 & 76 & 162 & 35 & 13 & 0 & 0,8 & 52,9 & 70,5 & 75,1 & 1,32 \\
280 & 6,0 & 228 & 168 & 42 & 14 & 1 & 0,9 & 61,2 & 81,0 & 75,6 & 1,32 \\
\hline
\end{tabular}


$\mathrm{O}$ cálculo do $\mathrm{P}$ aplicado para máxima eficiência micorrízica revelou, no conjunto das quatro variedades, valores entre 23 e $30 \mathrm{mg} \mathrm{dm}^{-3}$ (Tabela 2), o que corresponde a valores de 12 a $16 \mathrm{mg} \mathrm{dm}^{-3}$ de P disponível no solo (Mehlich-1). Nestas condições, o uso da inoculação de FMA nas variedades Baixinho de Santa Amália e Tainung no 1 promoveu valores de produção da parte aérea de até $86 \%$ da produção máxima calculada, nas plantas não-micorrizadas com doses bem maiores. O benefício de $\mathrm{P}$ foi maior nas variedades Sunrise Solo e Improved Sunrise Solo, enquanto as variedades Baixinho e Tainung apresentaram maior benefício micorrízico (Tabela 3). Disto resultou maior eficiência simbiótica nas variedades
Baixinho e Tainung, principalmente com o uso de G. margarita. A máxima resposta à micorriza ocorreu em teores de $\mathrm{P}$ disponível semelhantes em todas as variedades, o que indica ser uma característica sob maior controle da planta. Esse teor de P disponível deve ser influenciado tanto pelo tipo de solo, especificamente sua CMAP, como pelo extrator usado na sua análise, já que, diferentemente do método da Resina, o valor obtido pelo Mehlich-1 é dependente da capacidade-tampão do solo. A resposta à micorriza observada com relação ao mamoeiro é superior a outras fruteiras, como: abacate e manga (Silva \& Siqueira, 1991), banana (Declerck et al., 1995), maçã (Hoepfner et al., 1983) e abacaxi (Jaizme-Vega \&
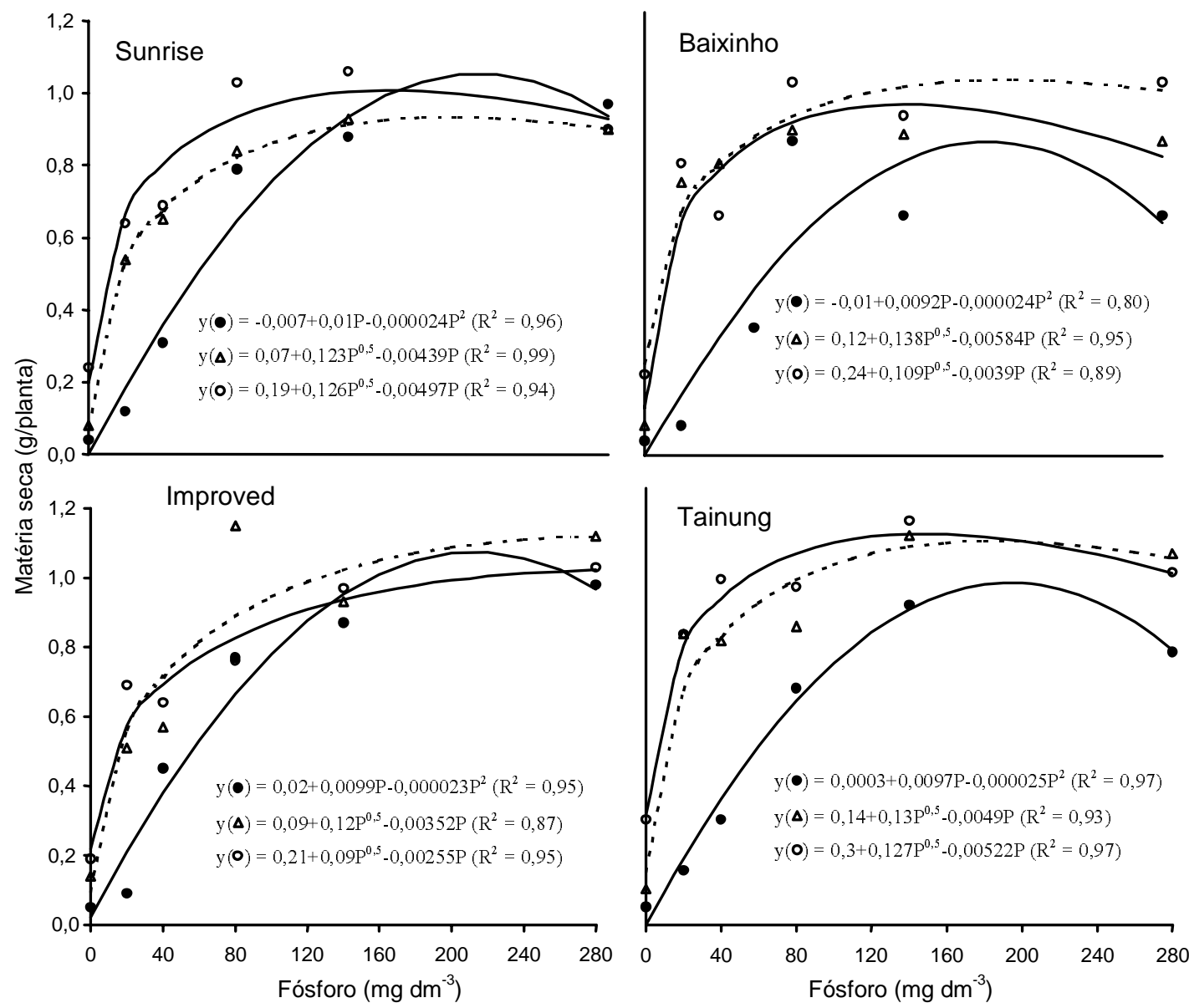

Figura 1. Produção de matéria seca de parte aérea de quatro variedades de mamoeiro sem inoculação ecom inoculação dos fungos micorrízicos G. clarum (৯) e G. margarita (O), em doses crescentes de P aplicadas ao solo. 
Azcon, 1991); estes resultados ainda são superiores aos obtidos com café (Saggin Júnior \& Siqueira, 1996), micorrizado com o mesmo isolado de G. clarum, embora, neste mesmo trabalho, um isolado de G. margarita tenha promovido eficiência de 97\% naquela cultura.

O ponto de cruzamento das curvas de produção de MSPA das plantas-controle e das micorrizadas, tido como valor T', só ocorreu nas variedades Sunrise
Solo e Improved Sunrise Solo (Figura 1). Em Sunrise Solo, o fungo G. margarita promoveu maior valor T' mas Improved Sunrise Solo foi mais responsivo a G. clarum, cuja eficiência ocorreu até a maior dose de $\mathrm{P}$ aplicada, sendo esse o valor T' (Tabela 2). Em relação às variedades Baixinho e Tainung as plantas inoculadas produziram maior biomassa em todas as doses de $\mathrm{P}$ para ambos os fungos, sendo o valor T' o limite superior do intervalo das doses utilizadas

Tabela 2. Doses de $\mathrm{P}$ aplicadas e P disponível no solo para promover a máxima eficiência micorrízica nas diferentes variedades de mamoeiro.

\begin{tabular}{|c|c|c|c|c|}
\hline Fungos & Sunrise Solo & Sunrise Solo, $72 / 12$ & Baixinho Sta. Amália & Tainung $\mathrm{n}^{\circ} 1$ \\
\hline & \multicolumn{4}{|c|}{ P aplicado $\left(\mathrm{mg} \mathrm{dm}^{-3}\right)$} \\
\hline Controle $^{(1)}$ & $67,0-90,5$ & $77,8-73,0$ & $111,0-106,5$ & $94,0-119,5$ \\
\hline G. clarum & 23,8 & 30,5 & 29,3 & 25,5 \\
\hline \multirow[t]{2}{*}{ G. margarita } & 25,5 & 27,0 & 23,9 & 25,5 \\
\hline & \multicolumn{4}{|c|}{ P disponível $\left(\mathrm{mg} \mathrm{dm}^{-3}\right)$} \\
\hline Controle $^{(1)}$ & $33,8-46,0$ & $39,3-36,9$ & $57,2-55,0$ & $47,9-62,7$ \\
\hline G. clarum & 12,7 & 16,0 & 15,4 & 13,5 \\
\hline G. margarita & 13,5 & 14,3 & 12,7 & 13,5 \\
\hline
\end{tabular}

(1)Primeiro e segundo valores correspondem ao $\mathrm{P}$ aplicado no tratamento controle não-inoculado, necessário para atingir produção equivalente aos tratamentos de inoculação com G. clarum e G. margarita, respectivamente.

Tabela 3. Benefício micorrízico, eficiência simbiótica e valor T' para variedades de mamoeiro submetidas à inoculação de G. clarum ou G. margarita, em doses crescentes de P aplicadas ao solo.

\begin{tabular}{|c|c|c|c|c|c|}
\hline \multirow{2}{*}{ Fungo } & \multicolumn{2}{|c|}{ Benefício } & \multirow{2}{*}{$\begin{array}{c}\text { Eficiência simbiótica } \\
(\%)\end{array}$} & \multirow{2}{*}{$\begin{array}{l}\text { Classificação } \\
\text { de eficiência }{ }^{(1)}\end{array}$} & \multirow{2}{*}{$\begin{array}{l}\text { Valor T' } \\
\left(\mathrm{mg} \mathrm{dm}^{-3}\right)\end{array}$} \\
\hline & (adimensional) & $\begin{array}{l}\text { Micorrízico } \\
\text { ional) }\end{array}$ & & & \\
\hline & & & Sunrise Solo & & \\
\hline Controle & 218,11 & & & & \\
\hline G. clarum & & 27,0 & 12,4 & Baixa & 128 \\
\hline \multirow[t]{2}{*}{ G. margarita } & & 44,0 & 20,2 & Baixa & 165 \\
\hline & \multicolumn{5}{|c|}{ Sunrise Solo - Line $72 / 12$} \\
\hline Controle & 224,07 & & & & \\
\hline G. clarum & & 39,6 & 17,7 & Baixa & 280 \\
\hline \multirow[t]{2}{*}{ G. margarita } & & 27,0 & 12,0 & Baixa & 132 \\
\hline & \multicolumn{5}{|c|}{ Baixinho de Sta. Amália } \\
\hline Controle & 177,6 & & & & \\
\hline G. clarum & & 62,1 & 35,0 & Alta & 280 \\
\hline \multirow[t]{2}{*}{ G. margarita } & & 81,0 & 45,6 & Alta & 280 \\
\hline & \multicolumn{5}{|c|}{ Tainung $n^{\circ} 1$} \\
\hline Controle & 197,4 & & & & \\
\hline G. clarum & & 68,3 & 34,6 & Alta & 280 \\
\hline G. margarita & & 79,5 & 40,3 & Alta & 280 \\
\hline
\end{tabular}

${ }^{(1)}$ Saggin Júnior \& Siqueira (1995) 
(280 $\mathrm{mg} \mathrm{dm}^{-3}$ de $\mathrm{P}$ aplicado e $228 \mathrm{mg} \mathrm{dm}^{-3}$ de $\mathrm{P}$ disponível).

Baseando-se na concepção de Habte \& Manjunath (1991) que relaciona dependência à condição de $\mathrm{P}$ no solo para as plantas - deficiente, máximo benefício da micorriza, e ótima - as variedades Baixinho e Tainung $\mathrm{n}-1$ se enquadraram como extremamente dependentes da micorriza, e Sunrise Solo e Improved Sunrise Solo estão num patamar inferior, mas ainda seriam altamente dependentes. Esta categorização corrobora os valores encontrados de T' (Janos, 1988) e de eficiência simbiótica (Saggin Júnior \& Siqueira, 1995).

Embora diminuindo com o aumento das doses de $\mathrm{P}$, a resposta à inoculação se manteve positiva no Baixinho e no Tainung, o que indica serem as variedades mais dependentes da micorriza, de acordo com o valor T', corroborando, assim, a classificação de Habte \& Manjunath (1991) e a eficiência simbiótica (Saggin Júnior \& Siqueira, 1995). A determinação do benefício micorrízico, tomando-se por base toda a amplitude de resposta, é mais completa, e está, provavelmente, menos sujeita a imprecisões, refletindo, portanto, o grau de eficiência (Janos, 1988), já que esta característica está intrinsecamente relacionada com a disponibilidade de $\mathrm{P}$ no solo. Entretanto, o cálculo do efeito do fungo no ponto de máxima eficiência micorrízica apresentou elevada correlação $\left(\mathrm{r}=0,999^{* *}\right)$ com a eficiência simbiótica estabelecida pelo método gráfico. Ou seja, o ponto de máxima eficiência micorrízica se mostrou bom indicador do grau de dependência de cada variedade de mamoeiro testada.

Quanto à produção de raízes em plantas nãomicorrizadas, o que constitui uma característica intrínseca, as variedades apresentaram um comportamento bastante distinto (Figura 2), contrastando com a produção da parte aérea, que foi semelhante em todas as variedades. Em todas as doses de P aplicadas, as variedades Tainung $\mathrm{n} \mathrm{o} 1$ e Baixinho apresentaram os menores valores de comprimento radicular sendo que, entre ambas, a menor resposta foi da variedade Tainung $\mathrm{n}-1$. A maior eficiência simbiótica constatada nas variedades Baixinho e Tainung foi o reflexo conjunto de um menor benefício de $\mathrm{P}$ e um maior benefício micorrízico. Este menor benefício do $\mathrm{P}$, resulta, provavelmente, da menor capacidade de produção de raízes destas variedades. Ou seja, as variedades apresentam capacidade semelhante de produção de parte aérea, mas esta capacidade só é plenamente expressa com a inoculação. Por outro lado, a produção de raízes é inferior nessas varieda-

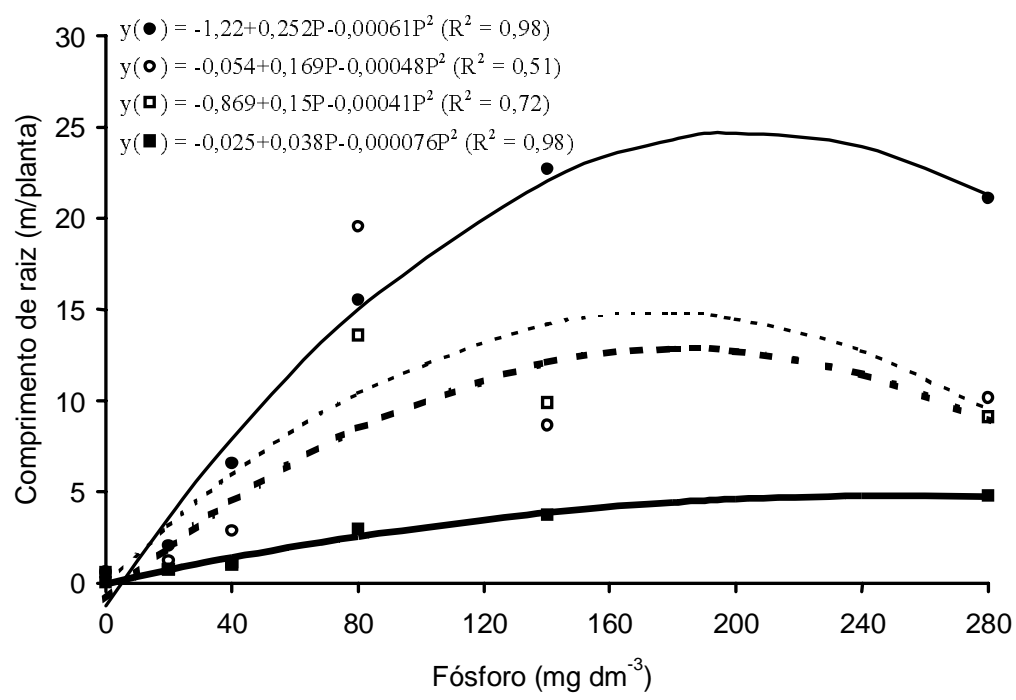

Figura 2. Comprimento de raiz das variedades de mamoeiro Sunrise (()), Improved (O), Baixinho ( $\square$ ) e Tainung ( $\square$ ), em doses crescentes de P aplicadas ao solo. 
des, e, de acordo com Koide (1991), é uma das características que confere maior resposta da planta à micorrização, pois reduz o suprimento de $\mathrm{P}$ à planta. Isto evidencia a importância do melhoramento na expressão da dependência micorrízica. A variedade Tainung $\mathrm{n}^{\mathrm{o}} 1$ é resultante do cruzamento de um tipo de mamão da Costa Rica, de polpa vermelha, com Sunrise Solo (Dantas, 1999), enquanto que o Baixinho de Santa Amália é resultante de mutação em Sunrise Solo, detectado no Espírito Santo.

O padrão de colonização de G. clarum foi semelhante nas quatro variedades, aumentando com a aplicação de $20 \mathrm{mg} \mathrm{dm}^{-3}$ de $\mathrm{P}$, e diminuindo nas doses subseqüentes (Figura 3). Considerando-se todas as doses de $\mathrm{P}$, a variedade Tainung $\mathrm{n} \div 1$ apresentou menor taxa de colonização por este fungo. Em todas as variedades, o G. clarum promoveu maior porcentual de colonização que G. margarita, e atingiu maiores valores em Sunrise Solo. A colonização por G. margarita aumentou com a dose de $20 \mathrm{mg} \mathrm{dm}^{-3}$ de P nas variedades Improved Sunrise Solo e Tainung, diferentemente do que ocorreu com a Sunrise Solo e com a Baixinho. Nos dois fungos, principalmente no G. margarita, o aumento das doses de P acima de $20 \mathrm{mg} \mathrm{dm}^{-3}$ reduziu a colonização, em todas as variedades, tendendo a zero na maior dose de $\mathrm{P}$ $\left(280 \mathrm{mg} \mathrm{dm}^{-3}\right)$. A diferença de colonização entre as espécies fúngicas não influenciou no benefício micorrízico promovido por cada uma, demonstrando, assim, que a medição de colonização interna total pode não refletir o grau de resposta da planta, pois nem sempre representa características importantes para o efeito do fungo como a capacidade de produção de micélio externo. Entretanto, na faixa de má-

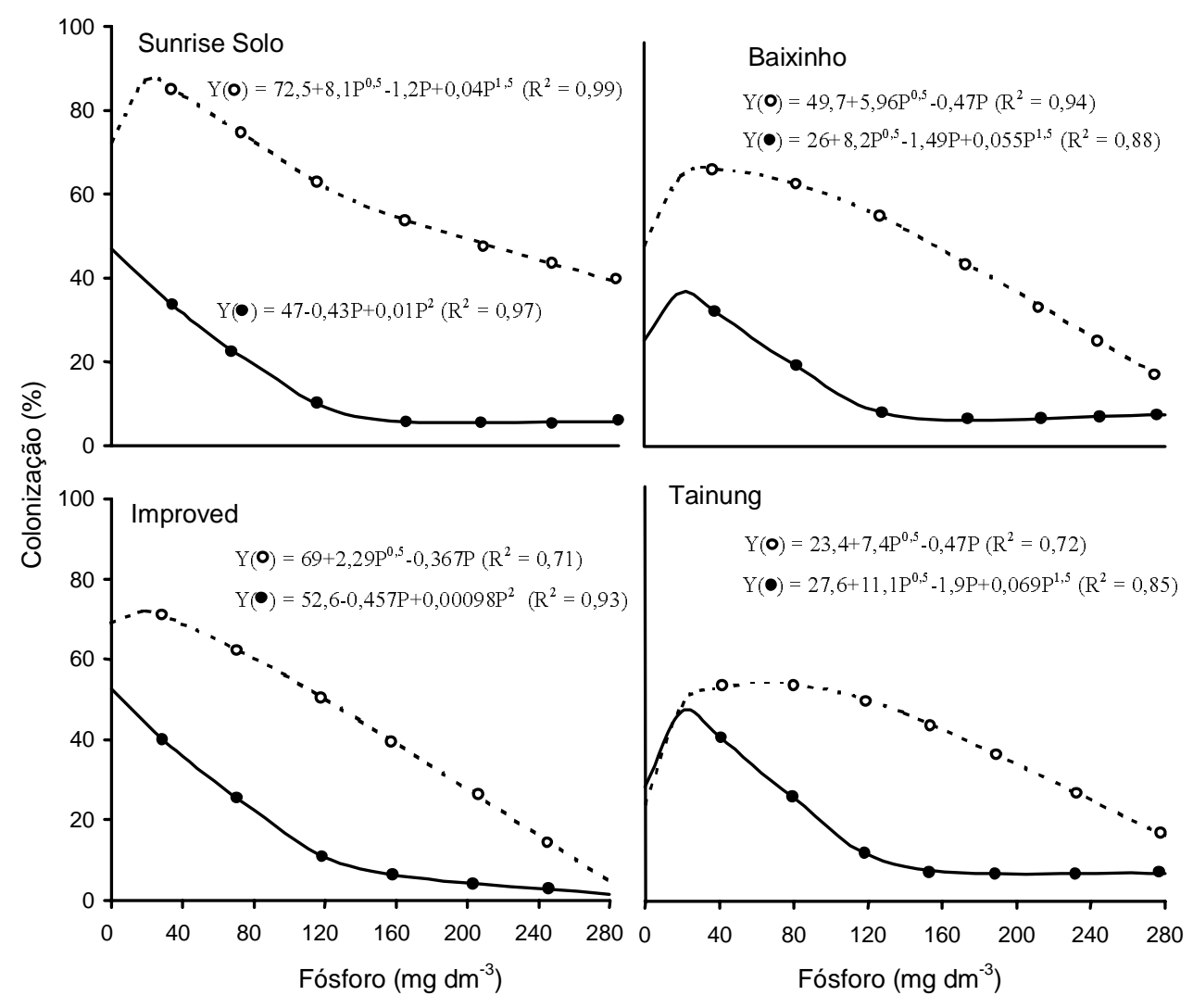

Figura 3. Porcentagem de colonização micorrízica de quatro variedades de mamoeiro, com inoculação dos fungos micorrízicos G. clarum (O) e G. margarita (•), em doses crescentes de P aplicadas ao solo. 
xima eficiência micorrízica, ocorreram os maiores porcentuais de colonização; isto indica a importância de se ter a combinação de doses de P suficientemente baixas, para propiciar um elevado porcentual de colonização micorrízica e sua expressão. Em condições de campo, a colonização exibida por G. clarum seria importante, pela possibilidade de manter maior taxa em doses mais elevadas de P. Doses de P acima de $140 \mathrm{mg} \mathrm{dm}^{-3}$ (76 $\mathrm{mg} \mathrm{dm}^{-3}$ de $\mathrm{P}$ disponível) reduzem a colonização a taxas muito baixas, sem promover incremento na produção de parte aérea.

A concentração de $\mathrm{P}$ na parte aérea das plantas aumentou com o aumento nas doses de $\mathrm{P}$, em todas as variedades, independentemente da inoculação (Figura 4). Semelhante ao padrão observado na produ- ção de matéria seca da parte aérea (Figura 1), a inoculação promoveu um grande incremento nos teores de $\mathrm{P}$ na planta nas menores doses de $\mathrm{P}$ aplicadas no solo, ficando demonstrada, assim, uma relação entre a absorção de $\mathrm{P}$ e o desenvolvimento da planta no intervalo das doses de 0 a $140 \mathrm{mg} \mathrm{dm}^{-3}$. Em todas as variedades, mas mais acentuado em Baixinho e Tainung, existe um ponto na curva de resposta de teores de $\mathrm{P}$, na planta, ao $\mathrm{P}$ do solo, a partir do qual os valores das plantas micorrizadas são inferiores aos valores das plantas não-micorrizadas. O uso de doses de $\mathrm{P}$ acima desses valores resulta em pequenos incrementos na produção de matéria seca da parte aérea.
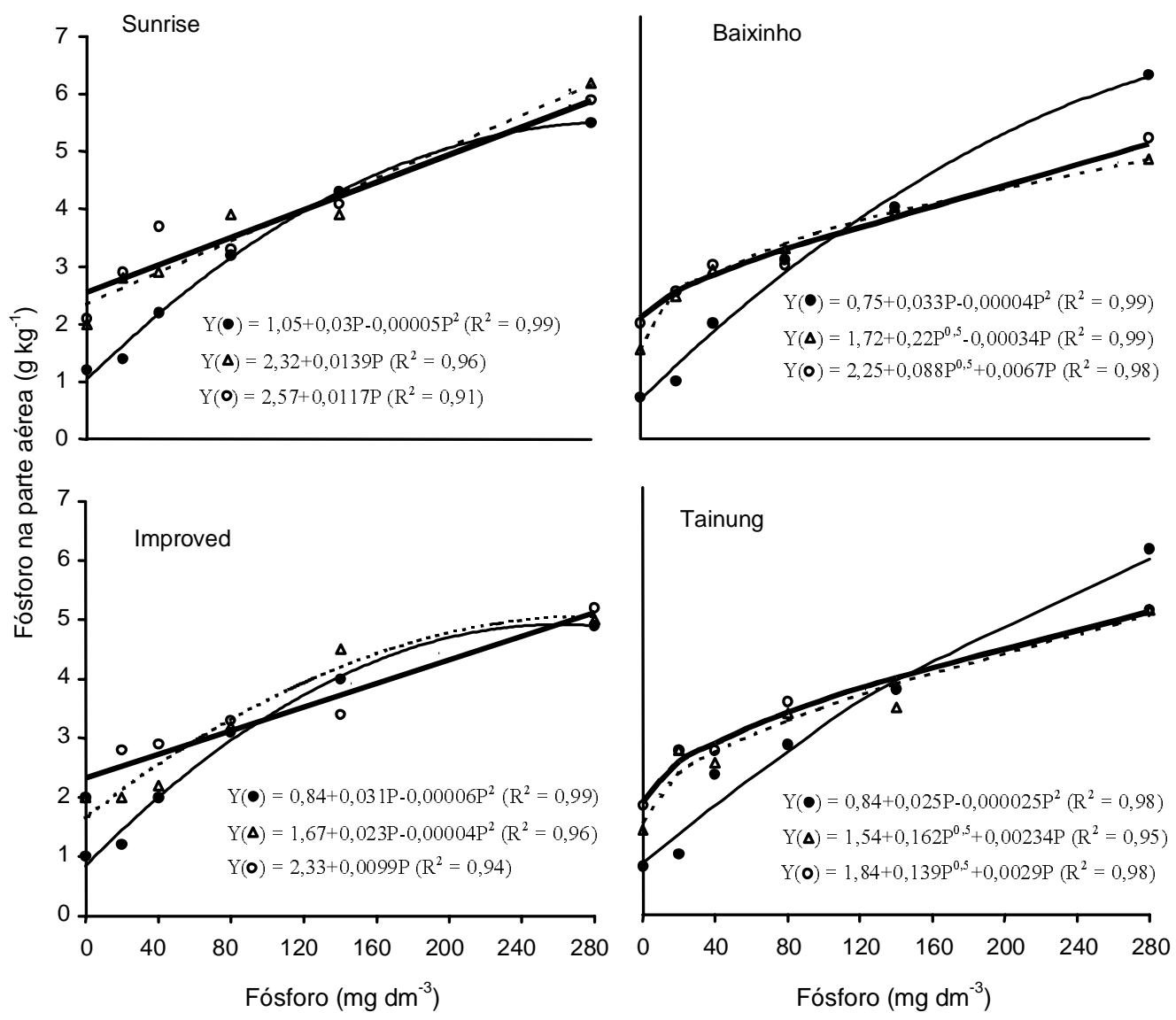

Figura 4. Teores de P da parte aérea de quatro variedades de mamoeiro sem inoculação $(\bullet)$ e com inoculação dos fungos micorrízicos G. clarum (\) e G. margarita (O), em doses crescentes de P aplicadas ao solo. 


\section{Conclusões}

1. A máxima eficiência micorrízica do mamoeiro situa-se na faixa de 12 a $16 \mathrm{mg} \mathrm{dm}^{-3}$ de P disponível.

2. O uso da inoculação de fungos micorrízicos nas variedades Baixinho de Santa Amália e Tainung no 1 reduz em até sete vezes a necessidade de P no solo, para se atingir a máxima produção de parte aérea.

3. O mamoeiro apresenta alto grau de dependência à micorriza, e é classificado de acordo com a variedade na seguinte ordem: Baixinho de Santa Amália $=$ Tainung $\mathrm{n}^{\mathrm{o}} 1>$ Sunrise Solo $=$ Improved Sunrise Solo.

4. No mamoeiro, a dependência micorrízica relaciona-se com a capacidade da variedade em produzir raízes.

\section{Referências}

AMBLER, J. R.; YOUNG, J. L. Techniques for determining root length infected by vesicular-arbuscular mycorrhizae. Soil Science Society of America Journal, Madison, v. 4, p. 551-556, 1977.

AMIJEE, F.; STRIBLEY, D. P.; TINKER, P. B. The development of endomycorrhizal root systems: effects of soil phosphorus and fungal colonization on the concentration of soluble carbohydrates in roots. New Phytologist, Cambridge, Inglaterra, v. 123, n. 2, p. 297-306, 1993

AULER, P. A. M. Desenvolvimento inicial do mamoeiro (Carica papaya $\mathrm{L}$.) relacionado à disponibilidade de fósforo no solo e à colonização pelo fungo micorrízico vesículo-arbuscular Glomus macrocarpum. Botucatu: Unesp, 1995. 94 p. Dissertação de Mestrado.

CLEMENT, C. R.; HABTE, M. Genotypic variation in vesicular-arbuscular mycorrhizal dependence of the pejibaye palm. Journal of Plant Nutrition, New York, v. 18, n. 9, p. 1907-1916, 1995

DANTAS, J .L. L. Cultivares. In: SANCHES, N. F; DANTAS, J. L. L. (Coord.). O cultivo do mamão. Cruz das Almas: Embrapa-CNPMF, 1999. p. 6-8. (Circular Técnica, 34).

DECLERCK, S.; PLENCHETTE, C.; STRULLU, D. G. Mycorrhizal dependency of banana (Musa acuminata, AAA group) cultivar. Plant and Soil, Dordrecht, v. 176, n. 1, p. $183-187,1995$.
HABTE, M.; MANJUNATH, A. Categories of vesiculararbuscular mycorrhizal dependency of host species. Mycorrhiza, Berlin, v. 1, n. 1, p. 3-12, 1991.

HOEPFNER, E. F.; KOCH, B. L.; COVEY, R. P. Enhancement of growth and phosphorus concentrations in apple seedlings by vesicular-arbuscular mycorrhizae Glomus mosseae. Journal of the American Society for Horticultural Science, Alexandria, v. 108, n. 2, p. $207-209,1983$.

JAIZME-VEGA, M. C.; AZCON, R. Effect of vesiculararbuscular mycorrhizal fungi on pineapple (Ananas comosus (L.) Merr.) in the Canary Islands. Fruits, Paris, v. 46, n. 1, p. $47-50,1991$

JANOS, D. P. Mycorrhiza applications in tropical forestry are temperate-zone approaches appropriate? In: NG, F. S. P. (Ed.). Trees and mycorrhiza. Kuala Lumpur: Forest Research Institute, 1988. p. 133-188

KOIDE, R. T. Nutrient supply, nutrient demand and plant response to mycorrhizal infection. New Phytologist, Cambridge, Inglaterra, v. 117, n. 3, p. 365-386, 1991

MANJUNATH, A.; HABTE, M. Root morphological characteristics of host species having distinct mycorrhizal dependence. Canadian Journal of Botany, Ottawa, v. 69, n. 5, p. 671-676, 1991.

MOHANDAS, S. Effect of VAM inoculation on plant growth, nutrient level and root phosphatase activity in papaya (Carica papaya cv. Coorg honey dew). Fertilizer Research, Dordrecht, v. 31, n. 3, p. 263-267, 1992.

NEWMAN, E. I. A method of estimating the total length of root in a sample. Journal of Applied Ecology, Oxford, v. 3, n. 2 , p. $139-145,1966$

OLIVEIRA, A. A. R.; WEBER, B.; SILVA, A. C. G. M. Micorrização e crescimento de porta-enxertos de citros em função de inóculos micorrízicos vesículo-arbusculares. Pesquisa Agropecuária Brasileira, Brasília, v. 27, n. 7, p. 1049-1056, jul. 1992

RAMIREZ, B. N.; MITCHELL, D. J.; SCHENCK, N. C. Establishment and growth effects of three vesiculararbuscular mycorrhizal fungi on papaya. Mycologia, New York, v. 67, n. 10, p. 1039-1041, 1975

SAGGIN JÚNIOR, O. J.; SIQUEIRA, J. O. Avaliação da eficiência simbiótica de fungos endomicorrízicos para o cafeeiro. Revista Brasileira de Ciência do Solo, Campinas, v. 19, n. 2, p. 221-228, 1995

SAGGIN JÚNIOR, O. J.; SIQUEIRA, J. O. Micorrizas arbusculares em cafeeiro. In: SIQUEIRA, J. O. (Ed.). 
Avanços em fundamentos e aplicação de micorrizas Lavras: Ufla, 1996. p. 203-254

SIEVERDING, E. Vesicular-arbuscular mycorrhiza management in tropical agrosystems. Eschborn: Bremer, 1991. $371 \mathrm{p}$

SILVA, L. F. C.; SIQUEIRA, J. O. Crescimento e teores de nutrientes de mudas de abacateiro, mangueira e mamoeiro sob influência de diferentes espécies de fungos micorrízicos vesículo-arbusculares. Revista Brasileira de Ciência do Solo, Campinas, v. 15, n. 3, p. 283-288, 1991

TRINDADE, A. V. Micorrizas arbusculares em mamoeiro. Lavras: Ufla, 1998. 177 p. Tese de Doutorado.

WEBER, O. B.; AMORIM, S. M. C. Adubação fosfática e inoculação de fungos micorrízicos vesículo-arbusculares em mamoeiro 'Solo'. Revista Brasileira de Ciência do Solo, Campinas, v. 18, n. 2, p. 187-191, 1994. 